\title{
A SHARP ESTIMATE ON THE BERGMAN KERNEL OF A PSEUDOCONVEX DOMAIN
}

\author{
SIQI FU
}

(Communicated by Eric Bedford)

\begin{abstract}
In this note we obtain a sharp estimate of the Bergman kernels near $\mathscr{C}^{2}$ boundary points of pseudoconvex domains by induction on the dimension and a theorem of Ohsawa-Takegoshi.
\end{abstract}

Let $\Omega$ be a bounded domain in $\mathbb{C}^{n}$, and let $A^{2}(\Omega)$ be the set of holomorphic functions on $\Omega$ in $L^{2}(\Omega)$. The Bergman kernel function of $\Omega$ on the diagonal can be defined by

$$
K_{\Omega}(z, \bar{z})=\sup \left\{|f(z)|^{2} ; f \in A^{2}(\Omega),\|f\|_{2} \leq 1\right\} .
$$

For general properties of the Bergman kernel, we refer the readers to Bergman's book [B]. The following theorem was first proved by Pflug [P]:

Theorem (Pflug). If $\Omega$ is a bounded pseudoconvex domain in $\mathbb{C}^{n}$ with $\mathscr{C}^{2}$ boundary near $p \in \partial \Omega$, then for each $\varepsilon>0$, there exist a constant $C>0$ and a neighborhood $U$ of $p$ such that

$$
K_{\Omega}(z, \bar{z}) \geq C \frac{1}{d^{2-\varepsilon}(z)}
$$

for all $z \in U \cap \Omega$, where $d(z)=\operatorname{dist}(z, \partial \Omega)$.

A generalization of this theorem was given by Catlin [C, Lemma 1]. The proofs given by Pflug and Catlin were based on the deep results of Skoda [S] on the $L^{2}$ estimate of $\bar{\partial}$ solutions.

In this note, we show that the $\varepsilon$ in the previous theorem can be eliminated. This result is implicit in the work of Ohsawa-Takegoshi. The purpose of this note is to draw attention to it.

Theorem. If $\Omega$ is a bounded pseudoconvex domain in $\mathbb{C}^{n}$ with $\mathscr{C}^{2}$ boundary near $p \in \partial \Omega$, then there exist a constant $C>0$ and a neighborhood $U$ of $p$ such that

$$
K_{\Omega}(z, \bar{z}) \geq C \frac{1}{d^{2}(z)} \quad \text { for all } z \in U \cap \Omega .
$$

Received by the editors August 6, 1993.

1991 Mathematics Subject Classification. Primary 32H10.

Key words and phrases. Bergman kernel, pseudoconvex domain. 
We shall prove the theorem by induction on the dimension and the deep theorem of Ohsawa-Takegoshi [O-T, p. 197].

Proof of the Theorem. Let $U$ be a tubular neighborhood of $\partial \Omega$ near $p$ such that for all $z \in U \cap \Omega$ there exists unique projection to the boundary $\pi(z)$ such that $d(z)=\operatorname{dist}(z, \pi(z))$. Let $n_{\pi(z)}$ be the outward normal direction at $\pi(z)$. We may assume that $\Omega$ is disjoint from the ball with center at $\pi(z)+\delta n_{\pi(z)}$ and radius $\delta$ for some $\delta>0$ independent of $z \in U \cap \Omega$.

Now for each $z_{0} \in U \cap \Omega$, after a translation and a unitary transformation, we may assume that $\pi\left(z_{0}\right)$ is the origin and $\operatorname{Re} z_{1}$-axis is the outward normal direction at $\pi\left(z_{0}\right)$. Hence $z_{0}=\left(-d\left(z_{0}\right), 0, \ldots, 0\right)$. If we set $H_{1}=$ $\left\{\left(z_{1}, z_{2}, \ldots, z_{n}\right) \in \mathbb{C}^{n} ; z_{n}=0\right\}$, then there exists $f \in A^{2}\left(\Omega \cap H_{1}\right)$ with $\|f\|_{2} \leq 1$ such that $K_{\Omega \cap H_{1}}\left(z_{0}, \bar{z}_{0}\right)=\left|f\left(z_{0}\right)\right|^{2}$. By the above-mentioned theorem of Ohsawa-Takegoshi, there exists $F \in A^{2}(\Omega)$ such that $\|F\|_{2}^{2} \leq C_{1}$, $\left.F\right|_{\Omega \cap H_{1}}=f$ for some constant $C_{1}>0$ depending only on the diameter of $\Omega$. Therefore,

$$
K_{\Omega}\left(z_{0}, \bar{z}_{0}\right) \geq \frac{1}{C_{1}} K_{\Omega \cap H_{1}}\left(z_{0}, \bar{z}_{0}\right) .
$$

Since the component of $\Omega \cap H_{1}$ containing $z_{0}$ is also a pseudoconvex domain, by induction on the dimension, we obtain

$$
K_{\Omega}\left(z_{0}, \bar{z}_{0}\right) \geq \frac{1}{C_{1}^{\prime}} K_{\Omega \cap H_{n-1}}\left(z_{0}, \bar{z}_{0}\right),
$$

for some $C_{1}^{\prime}>0$ depending only on diameter of $\Omega$, where $H_{n-1}=\left\{\left(z_{1}, z_{2}\right.\right.$, $\left.\left.\ldots, z_{n}\right) \in \mathbb{C}^{n} ; z_{2}=z_{3}=\cdots=z_{n}=0\right\}$. However, by our choices of the neighborhood $U$ and the coordinate system, we know that $\Omega \cap H_{n-1} \subset D_{\delta}=$ $\left\{\left(z_{1}, z_{2}, \ldots, z_{n}\right) \in \mathbb{C}^{n} ;\left|z_{1}-\delta\right|>\delta, z_{2}=\cdots=z_{n}=0\right\}$. Therefore,

$$
K_{\Omega \cap H_{n-1}}\left(z_{0}, \bar{z}_{0}\right) \geq K_{D_{\delta}}\left(z_{0}, \bar{z}_{0}\right) \text {. }
$$

On the other hand, it is clear that there exists $C_{2}>0$, depending only on $\delta$ such that

$$
K_{D_{\delta}}\left(z_{0}, \bar{z}_{0}\right) \geq C_{2} \frac{1}{d^{2}\left(z_{0}\right)} .
$$

Combining (2), (3), and (4), we conclude the proof of the theorem.

\section{REFERENCES}

[B] S. Bergman, The kernel function and conformal mapping, Amer. Math. Soc., Providence, RI, 1970.

[C] D. Catlin, Necessary conditions for subellipticity of the $\bar{\partial}$-Neumann problem, Ann. of Math. (2) 117 (1983), 147-171.

[O-T] T. Ohsawa and K. Takegoshi, On the extension of $L^{2}$ holomorphic functions, Math. Z. 195 (1987), 197-204.

[P] P. Pflug, Quadratintegrable holomorphische Funktionen und die Serre-Vermutung, Math. Ann. 216 (1975), 285-288.

[S] H. Skoda, Application des techniques $L^{2}$ à la théorie des ideaux d'un algèbre de fonctions holomorphes avec poids, Ann. Sci. École Norm. Sup. (4) 5 (1972), 545-580.

Department of Mathematics, Washington University, St. Louis, Missouri 63130

E-mail address: sfu@math.wustl.edu 